\section{ORIGINAL RESEARCH}

\author{
R. Agid \\ R.A. Willinsky \\ S.-K. Lee \\ K.G. TerBrugge \\ R.I. Farb
}

\title{
Characterization of Aneurysm Remnants after Endovascular Treatment: Contrast-Enhanced MR Angiography versus Catheter Digital Subtraction Angiography
}

\begin{abstract}
BACKGROUND AND PURPOSE: A substantial percentage of coiled aneurysms are associated with persistent filling of an aneurysmal component due to incomplete initial treatment or re-growth. Traditionally follow-up of coiled aneurysms has consisted of repeated intra-arterial cerebral catheter angiography, an invasive procedure with associated risks. Hence, many authors have advocated the use of non-invasive imaging techniques for this purpose. Our aim was to compare contrast-enhanced MR angiography (CE-MRA) with digital subtraction angiography (DSA) for depiction of aneurysmal remnants of coiled cerebral aneurysms.
\end{abstract}

\begin{abstract}
MATERIALS AND METHODS: Aneurysms coiled between September 2003 and October 2006 were retrospectively reviewed. We included patients meeting the following criteria: 1) residual/recurrent aneurysm measuring $2 \mathrm{~mm}$ or greater, and 2) CE-MRA and DSA performed no more than 60 days apart. Three readers were asked to determine which technique was superior for characterization of the aneurysmal remnant: CE-MRA, DSA, or indeterminate. Statistical analysis included most rule and $\kappa$ statistics.
\end{abstract}

\begin{abstract}
RESULTS: Of 232 patients who underwent coiling, 44 met the inclusion criteria (33 women and 11 men; 24-72 years of age). Sixteen patients had neck remnants and 28 had body remnants. The first study to identify the remnant was DSA in 35 patients and CE-MRA in 9. In 32 patients (32/44, 73\%), the readers indicated that CE-MRA was superior to DSA for remnant characterization. CE-MRA and DSA were thought to be equivalent in $7(16 \%)$, and DSA was preferred in $3(7 \%)$. Two cases $(5 \%)$ yielded ambiguous results. Of the 28 body remnants, 22 (78.6\%) were characterized by remnant protrusion into the coil mass: In 20 of these $(91 \%)$, the readers preferred CE-MRA over DSA, and in 2 cases $(9 \%)$, the techniques were thought to be equivalent.
\end{abstract}

CONCLUSION: In patients with known aneurysm remnants, CE-MRA is at least equivalent to DSA for characterization of aneurysmal remnants after coiling. Contrast filling within the coil mass was more clearly seen with CE-MRA than with DSA.

S ince the publication of the International Subarachnoid Aneurysm Trial, ${ }^{1}$ intracranial aneurysms are increasingly treated with endovascular coiling. A substantial percentage of coiled aneurysms are associated with persistent filling of an aneurysmal component either due to incomplete initial treatment or regrowth. ${ }^{1,2}$ Reconfiguration of the coil mass with time results in a recurrent aneurysmal neck or body remnant, ${ }^{3,4}$ which leads to reperfusion of the aneurysm, exposing the patient to a risk of rupture and rebleeding. ${ }^{1}$ For this reason, follow-up imaging (surveillance) of coiled aneurysms is important. When a considerable aneurysmal remnant is identified, recoiling is commonly advised. Traditionally this surveillance has consisted of repeated digital substraction angiography (DSA) in the months and years following the initial endovascular coiling. DSA is, however, an invasive procedure with well-known associated risks. ${ }^{5}$ Additional disadvantages include a short postprocedural hospital

Received January 23, 2008; accepted after revision March 19.

From the Department of Medical Imaging, Division of Neuroradiology, Toronto Western Hospital, University of Toronto, Toronto, Ontario, Canada.

Paper previously presented at: 9th Congress of the World Federation of Interventional and Therapeutic Neuroradiology, September 9-13, 2007; Beijing, China; won prize for best paper presented.

Please address correspondence to Ronit Agid, MD, Division of Neuroradiology, Department of Medical Imaging, Toronto Western Hospital, 399 Bathurst St, 3 McLaughling Wing, Room 425, Toronto, 0N, M5T 2S8, Canada; e-mail: ronit.agid@uhn.on.ca

DOI 10.3174/ajnr.A1124 stay, radiation exposure, allergic or nephrotoxic effects of iodinated contrast medium, and patient discomfort and expense. Hence, many authors have developed and advocated the use of noninvasive imaging techniques for the surveillance of aneurysm postcoiling. ${ }^{6-15}$

The accuracy and reliability of contrast-enhanced MR angiography (CE-MRA) to identify an aneurysm recurrence in coiled patients has been established. ${ }^{6,7,10,13}$ Although it is now recognized that CE-MRA is not devoid of risks, ${ }^{16,17}$ these risks are negligible and avoidable, compared with those of DSA. The purpose of this trial was to further evaluate the performance of CE-MRA for the depiction of the size and configuration of these aneurysmal remnants compared with DSA (the gold standard). This trial will help further define the role of noninvasive imaging in the surveillance of patients with previously coiled aneurysms.

\section{Materials and Methods}

This study was reviewed and approved by our institutional review board. All cases of coiled cerebral aneurysms performed in our institution between September 2003 and October 2006 were retrospectively reviewed. To be included in this study, patients had to harbor a residual or recurrent aneurysm (aneurysm remnant) $2 \mathrm{~mm}$ or greater (based on DSA). In addition, the patients were required to have both CE-MRA and DSA performed no more than 60 days apart. 

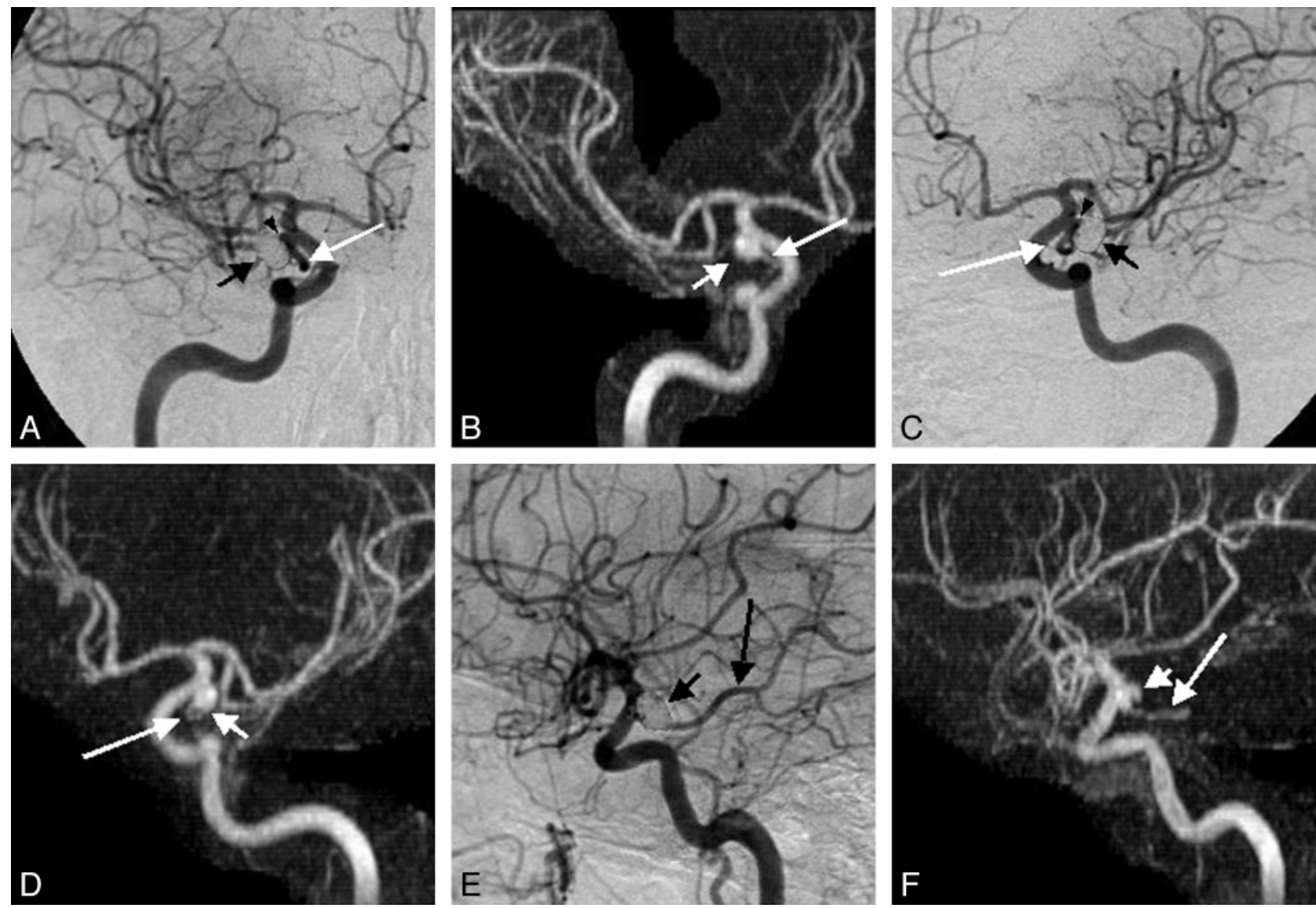

Fig 1. A 57-year-old woman, 10 months post-subarachnoid hemorrhage and endovascular coiling of a right PcomA aneurysm. $A, C$, and $E$, Multiple oblique images taken from a rotational DSA. Although a small neck residual is noted (arrowheads in $A$ and $C$ ), there is no evidence of contrast within the actual coil mass (short arrows in $A$, $C$, and $E$ ). Matching oblique MIP reconstructions of CE-MRA performed 29 days later reveal a much larger aneurysmal recurrence (short arrows in $B, D$, and $F$ ). This larger extent of recurrent aneurysm is difficult to appreciate on the rotational DSA images because it is surrounded by the coil mass, which attenuates the photons passing through it and creates the coil helmet effect. Note that the PcomA artery adjacent to the aneurysm is well demonstrated on both DSA and CE-MRA (long arrows in A-F).

\section{Imaging Methods}

MR Angiography. MR angiography (MRA) examinations were performed by using a $1.5 \mathrm{~T}$ MR imaging scanner (Signa HD; GE Healthcare, Milwaukee, Wis) with a standard head coil. The MRA examinations all included a CE-MRA by using gadobutrol (Gadovist; Bayer Schering Pharma, Berlin, Germany) injected at a rate of $1.5 \mathrm{~mL}$ per second to a total of $15 \mathrm{~mL}$, followed immediately by a flush of 30 $\mathrm{mL}$ of saline. The specific method in use at our institution is an autotriggered elliptic centric-ordered 3D gadolinium-enhanced MRA. ${ }^{18}$ This method of CE-MRA is similar to more ubiquitous forms of commercialized monitored or "fluorotriggered" CE-MRA used in many centers worldwide. The source images consist of an elliptic centricordered 3D fast echo-spoiled gradient-echo sequence oriented in the axial plane, with coverage from the foramen magnum to above the circle of Willis, with the following parameters: TR/TE, 3.7/1 ms; flip angle, $1.1^{\circ}$; fractional echo acquisition: FOV, $22 \times 22 \mathrm{~cm}$; matrix, $320 \times 320$; bandwidth, $62.5 \mathrm{kHz}$; section thickness, $1.0 \mathrm{~mm}$; flip angle, $30^{\circ}$; 76 sections resulting in a $7.6-\mathrm{cm}$-thick volume; and a scanning time of 2 minutes. Resultant voxel dimensions were $0.7 \times 0.5 \times$ $1.0 \mathrm{~mm}$ (this resolution was obtained without zero-filling techniques). 3D maximum intensity projection (MIP) reconstructions were available as well.

DSA. Catheter-based intra-arterial cerebral DSA was performed in all patients by using a dedicated biplane neuroangiography suite (LCN+, GE Medical Systems, Buc, France). All DSA examinations included frontal and lateral views with selective injection of the appropriate internal carotid or vertebral artery. All DSA examinations also included a subtracted rotational (spin) angiographic acquisition with selective arterial injection. The amounts of iodinated contrast medium (Omnipaque 300; GE Healthcare, Chalfont St. Giles, UK) injected for the anteroposterior and lateral views were the following: 5 $\mathrm{mL}$ per second to a total volume of $10 \mathrm{~mL}$ for the carotid artery and 3-4 $\mathrm{mL}$ per second to a total of 7-8 $\mathrm{mL}$ for the vertebral artery. For the rotational views, $4 \mathrm{~mL}$ per second to a total of $20 \mathrm{~mL}$ was used for the carotid artery and $3 \mathrm{~mL}$ to a total of $15 \mathrm{~mL}$ for the vertebral artery.

\section{Image Evaluation}

Three readers reviewed the consecutive CE-MRA and DSA studies for each patient. The 2 sets of images (CE-MRA and DSA) were presented to the readers at the same time. All readers were neuroradiologists practicing diagnostic and interventional neuroradiology. The CE-MRA studies presented to the readers included axial source images of the brain and MIP reconstructions of the appropriate artery associated with the coiled aneurysm. The DSA images provided to the readers included anteroposterior, lateral, and spin views of the specific vessel harboring the aneurysm. The readers were asked to determine which technique was superior for characterization of the aneurysmal remnant: CE-MRA, DSA, or indeterminate. Criteria for superiority included the ability to determine the size of remnants, the orientation, and the relationship to the parent vessel and coils. The readers were not required to record measurements but 

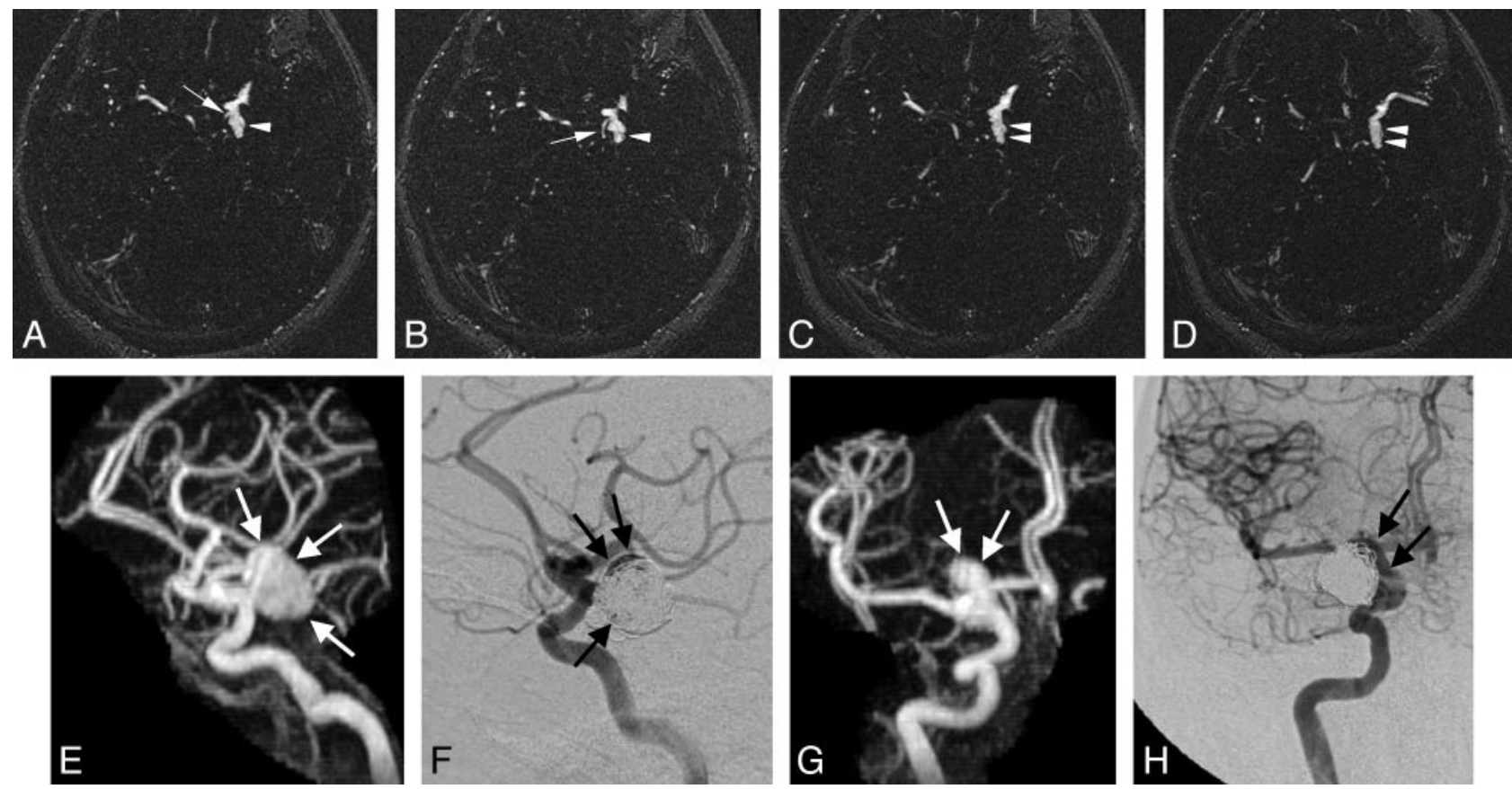

Fig 2. A 72-year-old woman, 14 months post-subarachnoid hemorrhage and endovascular coiling of a left PcomA aneurysm. $A-D$, Axial source images of CE-MRA. $E$ and $G$, Lateral ( $E)$ and anteroposterior (G) MIP reconstructions of CE-MRA demonstrate a large aneurysmal recurrence (arrowheads in $A-D$ and arrows in $E$ and G). F and $H$, DSA performed 2 months after the CE-MRA clearly shows a large aneurysmal recurrence (arrows), but the extent of this recurrence within and peripheral to the coil mass is difficult to appreciate. This aneurysm was recoiled with 9 coils. Note the excellent demonstration of the PcomA artery and its origin on the CE-MRA source images (arrows in $A$ and $B$ ).
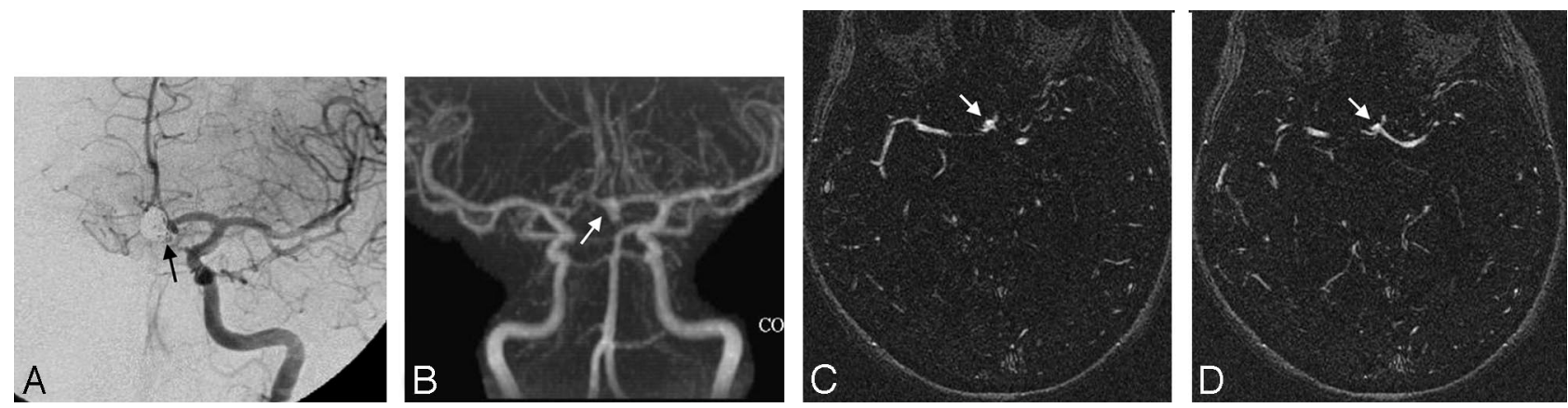

Fig 3. A 51-year-old woman presenting with subarachnoid hemorrhage. A, Final control left internal carotid DSA in the anteroposterior projection following endovascular coiling of an AcomA aneurysm. The coil mass is noted with only perhaps several faint tiny spots of suggested contrast within it (arrow). B, MIP reconstruction of CE-MRA in the anteroposterior projection performed 4 days later clearly shows a substantial aneurysmal body remnant filling with contrast (arrow) extending inferiorly. $C-D$, Axial source images of the CE-MRA show the remnant (arrows).

rather were asked to judge the size of the remnants and were encouraged to measure these remnants with electronic calipers where needed. The distinction between body and neck remnants in this study was performed according to the Roy et al classification. ${ }^{4}$

\section{Statistical Analysis}

For statistical analysis, we used most rule, in which the imaging technique (CE-MRA, DSA, or indeterminate) preferred by 2 of 3 readers was regarded as the technique of choice. The degree of agreement between the readers was determined by using an overall $\kappa$ statistic and following the guidelines suggested by Landis and Koch. ${ }^{19}$ In addition, the $95 \%$ confidence interval $(\mathrm{CI})$ was calculated to provide a range in which there is $95 \%$ confidence that the true value lies within the data we observed.

\section{Results}

Two hundred thirty-two patients who underwent aneurysm coiling with subsequent follow-up were identified in our data base. Forty-four of these were found to have substantial neck or body aneurysmal remnants ( $\geq 2 \mathrm{~mm}$ ) as well as CE-MRA and DSA performed in the requisite time window. These included 33 women and 11 men, 24-72 years of age (mean, 50.25). In 35 patients, DSA was performed before CE-MRA, and in 9 patients, CE-MRA was the earlier study revealing the aneurysmal remnant. All of the aneurysmal remnants were identified on the initial report of the CE-MRA (the corollary was also true for those who underwent DSA initially). The various locations of the aneurysms included in this study were the following: carotid termination in 3 patients, basilar tip in 11 patients, posterior communicating artery (PcomA) in 9 patients, anterior communicating artery (AcomA) in 10 patients, paraophthalmic region of the carotid artery in 3 patients, superior hypophyseal artery in 1 patient, vertebrobasilar junction in 2 patients, posterior inferior cerebellar artery in 1 patient, anterior choroidal artery in 1 patient, middle cerebral artery in 1 patient, cavernous carotid artery in 1 patient, and vertebral artery in 1 patient. 
Sixteen patients had aneurysmal neck remnants (16/44, $36.4 \%)$ and $28(28 / 44,63.6 \%)$ had body remnants. Twentytwo of the body remnants $(22 / 28,78.6 \%)$ were "helmet" style recurrences with protrusion of the residual aneurysm into the coil mass.

Our readers indicated that all 44 remnants were clearly identified on CE-MRA. In 32 of the 44 cases (73\%), the readers indicated that CE-MRA was superior to DSA for characterization of the remnant (Figs 1-3). CE-MRA and DSA were thought to be equivalent in $7(16 \%)$, and DSA was preferred in $3(7 \%)$. Two cases $(5 \%)$ yielded ambiguous results with the 3 readers selecting different preferences (no consensus [most] of opinion).

The overall $\kappa$ statistic for all 3 selections (MRA superior, DSA superior, or indeterminate) across all 3 readers was 0.49 (95\% CI, 0.35-0.62). With the guidelines suggested by Landis and Koch (1977), ${ }^{19}$ this result suggests that a moderate degree of agreement existed across the 3 raters. This result is statistically significant $(P=.0001)$, suggesting that the degree of agreement observed across the 3 raters is greater than we would expect to see by chance alone. Agreement was highest among cases where MRA was preferred. Among these cases, the agreement was 0.63 (95\% CI, 0.46-0.80, $P<.0001)$, which is considered a moderate degree of agreement.

\section{Helmet-Type Remnants}

Separate statistical analysis of the helmet-style remnants yielded yet a higher preference of MRA compared with DSA. In these cases, most readers indicated that MRA was better in $20(91 \%)$ (Figs 1, 3). In 17 of these 20 cases, there was unanimity among all 3 readers (ie, all readers chose MRA as superior to DSA). The remaining 2 cases (9\%) yielded ambiguous results with the 3 readers selecting a different preference (no consensus [most] of opinion).

\section{Discussion}

Because the long-term durability of endovascular treatment for aneurysms is yet to be proved and episodes of rebleeding following endovascular treatment do occur, ${ }^{1}$ surveillance of previously coiled aneurysms is mandatory. Although the use of MRA in this arena is increasing in popularity, ${ }^{6,7,13}$ DSA remains the perceived gold standard, and these patients are commonly (possibly in most cases) followed with periodic DSA. This classic clinical practice is reflected in the literature, where authors still question the ability of MRA to reveal aneurysmal remnants. ${ }^{20}$ Moreover, endovascular coiling as opposed to surgical clipping requires years of follow-up with repeated imaging; this fact has been cited as a potential discouragement for referral to endovascular treatment of aneurysms. $^{20}$ This discouragement is understandable when DSA, an invasive technique, is used exclusively as the surveillance technique.

Several studies have compared various MRA techniques with DSA with the ultimate goal of obviating repeated DSA in these patients. ${ }^{6-14,15,21}$ Earlier studies suggested nonenhanced 3D time of flight (TOF) MRA as the method of choice for following coiled aneurysms. Although some of these reports showed encouraging results for 3D TOF MRA, ${ }^{8,11,12,14,21}$ others did not have a similar experience because neck remnants and even recurrent aneurysms were repeatedly overlooked with this technique. ${ }^{6,13,15}$ Furthermore, a recent in vitro evaluation of TOF MRA showed that it underestimated the need for retreatment compared with DSA. ${ }^{9}$

Several authors have evaluated CE-MRA versus TOF MRA for coiled aneurysms, and most have reported superiority of CE-MRA. ${ }^{6,7,9,10,13}$ Most of the studies regarding CE-MRA conclude equivalency of CE-MRA and DSA in detecting aneurysmal remnants. Further support for the superiority of CEMRA over TOF MRA was recently re-emphasized for stentassisted coiling of aneurysms. ${ }^{10}$

Our observations in the present study only pertain to subjects with known aneurysmal remnants; thus, these observations should not be generalized to all postcoiling patients. However, the present study does further validate the previous reports and again illustrates that all substantial ( $>2 \mathrm{~mm})$ aneurysmal remnants (necks and bodies) noted on DSA are clearly identified on CE-MRA. Improvements in the CE-MRA technique since earlier publications are in contrast agents, $\mathrm{MR}$ imagers, field strength, and $3 \mathrm{D}$ reconstruction software. These ongoing refinements all potentially improve the ability to resolve tiny remnants; however, this study does not address the sensitivity of this technique for smaller remnants $(<2 \mathrm{~mm})$, which, in our opinion, are of less clinical importance.

Most interesting, the present study reveals that CE-MRA is likely superior to DSA for patients in whom aneurysm body remnants extend or invaginate into the coil mass. This can be explained by the radiodensity of the coil mass itself. In these instances, the coils that surround the aneurysmal remnant create an impenetrable radiodense helmet around the remnant, such that it is difficult to appreciate the remnant on routine DSA (anteroposterior, lateral, and rotational views). This feature is due to lack of photon penetration through the coil mass. MRA enjoys a distinct advantage over DSA for the visualization of this type of remnant because it does not have the helmet phenomenon and there is only minimal susceptibility artifact arising from platinum coils. ${ }^{22}$ Our readers preferred CE-MRA over DSA in 91\% of the helmet-type remnants.

The limitations of this study include the lack of a control group and the fact that all of the included patients were known to have an aneurysmal remnant. However, the aim of this study was not to evaluate the ability of CE-MRA to demonstrate such remnants but rather to compare CE-MRA to DSA for the characterization of known remnants. Also we recognize that presentation of both sets of images (DSA and CE-MRA) at the same time to the readers may have introduced a modicum of bias; nonetheless, it was thought that this was a realistic method to determine preference of technique.

Again, contrast-enhanced MR imaging carries a low risk of possible nephrogenic systemic fibrosis. ${ }^{16,17}$ To avoid this complication, one must exercise judgment when using gadolinium in patients with end stage kidney disease or renal functions approaching dialysis.

Our current practice at the Toronto Western Hospital is that all coiled patients are followed up primarily by CE-MRA. DSA is performed if CE-MRA depicts a large remnant or an enlarging remnant that requires retreatment. Postcoiling CEMRA is performed before patients' discharges, at 2 months (if ruptured), 6 months, 18 months, and every 2 years thereafter. This protocol is altered in case of a substantial or growing remnant. 


\section{Conclusion}

In subjects with known aneurysm remnants, CE-MRA is at least equivalent to DSA for characterization of aneurysm remnants after coiling. Contrast filling within the coil mass was more clearly seen with MRA than with DSA. This study further supports the use of CE-MRA as a noninvasive technique for the surveillance of coiled aneurysms.

\section{References}

1. Molyneux AJ, Kerr RS, Yu LM, et al. International subarachnoid aneurysm trial (ISAT) of neurosurgical clipping versus endovascular coiling in 2143 patients with ruptured intracranial aneurysms: a randomised comparison of effects on survival, dependency, seizures, rebleeding, subgroups, and aneurysm occlusion. Lancet 2005;366:809-17

2. Kole MK, Pelz DM, Kalapos P, et al. Endovascular coil embolization of intracranial aneurysms: important factors related to rates and outcomes of incomplete occlusion. J Neurosurg 2005;102:607-15

3. Vinuela F, Duckwiler G, Mawad M. Guglielmi detachable coil embolization of acute intracranial aneurysm: perioperative anatomical and clinical outcome in 403 patients. J Neurosurg 1997;86:475-82

4. Roy D, Milot G, Raymond J. Endovascular treatment of unruptured aneurysms. Stroke 2001;32:1998-2004

5. Willinsky RA, Taylor SM, TerBrugge K, et al. Neurologic complications of cerebral angiography: prospective analysis of 2,899 procedures and review of the literature. Radiology 2003;227:522-28. Epub 2003 Mar 13.

6. Farb RI, Nag S, Scott JN, et al. Surveillance of intracranial aneurysms treated with detachable coils: a comparison of MRA techniques. Neuroradiology 2005; $47: 507-15$

7. Gauvrit JY, Leclerc X, Caron S, et al. Intracranial aneurysms treated with Guglielmi detachable coils: imaging follow-up with contrast-enhanced MR angiography. Stroke 2006;37:1033-37. Epub 2006 Mar 9

8. Pierot L, Delcourt C, Bouquigny F, et al. Follow-up of intracranial aneurysms selectively treated with coils: prospective evaluation of contrast-enhanced MR angiography. AJNR Am J Neuroradiol 2006;27:744-49

9. Costalat V, Lebars E, Sarry L, et al. In vitro evaluation of 2D-digital subtraction angiography versus 3D-time-of-flight in assessment of intracranial cerebral aneurysm filling after endovascular therapy. AJNR Am J Neuroradiol 2006;27:177-84

10. Lubicz B, Levivier M, Sadeghi N, et al. Immediate intracranial aneurysm occlusion after embolization with detachable coils: a comparison between MR angiography and intra-arterial digital subtraction angiography. J Neuroradiol 2007;34:190-97

11. Yamada N, Hayashi K, Murao K, et al. Time-of-flight MR angiography targeted to coiled intracranial aneurysms is more sensitive to residual flow than is digital subtraction angiography. AJNR Am J Neuroradiol 2004;25:1154-57

12. Majoie CB, Sprengers ME, van Rooij WJ, et al. MR angiography at $3 T$ versus digital subtraction angiography in the follow-up of intracranial aneurysms treated with detachable coils. AJNR Am J Neuroradiol 2005;26:1349-56

13. Leclerc X, Navez JF, Gauvrit JY, et al. Aneurysms of the anterior communicating artery treated with Guglielmi detachable coils: follow-up with contrastenhanced MR angiography. AJNR Am J Neuroradiol 2002;23:1121-27

14. Okahara M, Kiyosue H, Hori Y, et al. Three-dimensional time-of-flight MR angiography for evaluation of intracranial aneurysms after endosaccular packing with Guglielmi detachable coils: comparison with 3D digital subtraction angiography. Eur Radiol 2004;14:1162-68. Epub 2004 Apr 21

15. Boulin A, Pierot L. Follow-up of intracranial aneurysms treated with detachable coils: comparison of gadolinium-enhanced 3D time-of-flight MR angiography and digital subtraction angiography. Radiology 2001;219:108-13

16. Grobner T. Gadolinium: a specific trigger for the development of nephrogenic fibrosing dermopathy and nephrogenic systemic fibrosis? Nephrol Dial Transplant 2006;21:1104-08. Epub 2006 Jan 23

17. Kuo PH, Kanal E, Abu-Alfa AK, et al. Gadolinium-based MR contrast agents and nephrogenic systemic fibrosis. Radiology 2007;242:647-49

18. Farb RI, McGregor C, Kim JK, et al. Intracranial arteriovenous malformations: real-time auto-triggered elliptic centric-ordered 3D gadolinium-enhanced MR angiography — initial assessment. Radiology 2001;220:244-51

19. Landis JR, Koch GG. The measurement of observer agreement for categorica data. Biometrics 1977;33:159-74

20. Findlay JM, Darsaut TE. Endovascular management of cerebral aneurysms: work in progress. Can J Neurol Sci 2007;34:1-2

21. Brunereau L, Cottier JP, Sonier CB, et al. Prospective evaluation of time-offlight MR angiography in the follow-up of intracranial saccular aneurysms treated with Guglielmi detachable coils. J Comput Assist Tomogr 1999;23:216-23

22. Hartman J, Nguyen T, Larsen D, et al. MR artifacts, heat production, and ferromagnetism of Guglielmi detachable coils. AJNR Am J Neuroradiol 1997; 18:497-501 